\title{
Suicidality in Bangladeshi Young Adults During the COVID-19 Pandemic: The Role of Behavioral Factors, COVID-19 Risk and Fear, and Mental Health Problems
}

\author{
Mohammed A Mamun (iD) 1,2 \\ Firoj al Mamun (D) ${ }^{1,2}$ \\ Ismail Hosen (D) I,2 \\ Mahmudul Hasan (iD) 1,3 \\ Abidur Rahman (D) ${ }^{1,3}$ \\ Ahsanul Mahbub Jubayar (D) I,3 \\ Zeba Maliha (iD) \\ Abu Hasnat Abdullah (D) 1,2 \\ Md Abedin Sarker ${ }^{1,2}$ \\ Humayun Kabir (D) ${ }^{1,5}$ \\ Avijit Sarker Jyoti (iD ${ }^{6}$ \\ Mark Mohan Kaggwa (iD ${ }^{7,8}$ \\ Md Tajuddin Sikder (iD ${ }^{2}$ \\ 'CHINTA Research Bangladesh, Dhaka, \\ Bangladesh; ${ }^{2}$ Department of Public \\ Health and Informatics, Jahangirnagar \\ University, Dhaka, Bangladesh; \\ ${ }^{3}$ Department of Biochemistry and \\ Molecular Biology, University of Rajshahi, \\ Rajshahi, Bangladesh; ${ }^{4}$ Bangabandhu \\ Sheikh Mujib Medical College, Faridpur, \\ Bangladesh; ${ }^{5}$ Department of Public \\ Health, North South University, Dhaka, \\ Bangladesh; ${ }^{6}$ Mugda Medical College, \\ Dhaka, Bangladesh; ${ }^{7}$ Department of \\ Psychiatry, Mbarara University of Science \\ \& Technology, Mbarara, Uganda; ${ }^{8}$ African \\ Centre for Suicide Prevention and \\ Research, Mbarara, Uganda
}

\begin{abstract}
Background: It is said that psychological stressors have risen during the COVID-19 pandemic, which may contribute to suicidality. A few studies were conducted investigating suicidality amid the first wave of the pandemic in Bangladesh, but none of these studies explored the predictive role of the suicidality-related factors (eg, behavior and health-related variables, COVID-19 risk, fear of COVID-19). Thus, this study aimed to investigate the prevalence of suicidality and the predictive models explaining suicidality variance among Bangladeshi young adults during the second wave of the pandemic.
\end{abstract}

Methods: An online-based cross-sectional survey was conducted during the second wave of the pandemic (between 1 and 13 April 2021; a year after the pandemic's inception in the country). The survey questionnaire collected information on socio-demographics, behavior and health-related variables, COVID-19 risk, fear of COVID-19, depression, anxiety, and suicidality. A total of 756 data were analyzed (male 59\%; mean age $22.24 \pm 4.39$ years), and hierarchical regression was performed considering suicidality as the dependent variable. A $p$-value $<0.05$ was set to be statistically significant with a $95 \%$ of confidence interval.

Results: About $8.2 \%$ reported having at least suicidal thoughts within the pandemic inception to survey time (one-year suicidal ideation). More specifically, $7.40 \%$ had only suicidal ideation, whereas $0.10 \%$ planned for suicide, $0.70 \%$ attempted suicide. None of the sociodemographic variables was significantly associated with suicidality. Taking drugs, performing less physical activity, poor self-reporting health condition, being comorbid, being at higher COVID-19 risk, having fear of COVID-19 infection, and suffering from depression and anxiety were significantly associated with a higher risk of suicidality. However, the final model including all of the studied variables explained a $17.1 \%(\mathrm{~F}=8.245, p<0.001)$ variance in terms of suicidality.

Conclusion: Although this study reported a lower severity of suicidality than prior Bangladeshi studies conducted during the first wave of the pandemic, a portion of people are still at risk of suicide and they should be given attention.

Keywords: COVID-19 suicide, psychological impact, suicide, self-harm, depression, fear of COVID-19, Bangladeshi youths

\section{Introduction}

The COVID-19 pandemic has turned into a public health emergency of international concern, later on, it was declared as a pandemic by the World Health Organization. The pandemic has led to overwhelming pressure on health-care
Correspondence: Mohammed A Mamun Tel +880 1738592653

Email mamunphi46@gmail.com 
systems due to the increasing number of cases and mortalities. At present, there is no accurate estimation about how long the situation will persist, how many people will get infected, or how long people's lives will get disrupted. This type of uncertainty decreases the quality of life including, higher panic towards the pandemic. ${ }^{1}$

From March 26, 2020, the government of Bangladesh declared a lockdown to suppress the spread of the virus. ${ }^{2}$ As a result, due to social distancing, all types of public activities including, educational institutes were closed down, along with imposing restrictions related to quarantine and isolation for suspected and confirmed cases, respectively. ${ }^{2}$ Restricted movements help reduce viral infection transmission, but such restrictions can lead to deteriorating mental health by reducing movement and physical activity. ${ }^{3-6}$ Along with this, because of other pandemic effects, a considerable proportion of people have been reported mentally affected. ${ }^{7,8}$ The countrywide lockdown hampered all sorts of economic activities, which led to higher economic constrictions. In line with the situation, the country was predicted to decline by $1.10 \%$ in its GDP, and about 9-million people to lose their jobs. ${ }^{9}$ Unemployment and economic crisis are linked to suicide occurrences in the country; that is, poverty and financial distress caused all but one COVID-19 related suicide cases occurred in the first 3-week of April 2020. ${ }^{10}$ Another study reported $46 \%$ of the suicide casualties to be unemployment-related economic issues turned by the lockdown, whereas family conflicts, parental abuse, parental dispute, xenophobia, social negligence and stigma, lack of access to treatment, fear, and panic, mental health problems, etc., were also accused of suicide stressors. ${ }^{11}$

Leaune et al ${ }^{12}$ recently reviewed the factors associated with suicide during the emerging viral disease outbreaks whereas fear of contacting the virus was reported being the most significant factor, along with others such as being socially isolated or disconnected, having everyday life disrupted, experiencing burden related to long-term illnesses, having unusual concerns about hygiene, being more reluctant to receive medical care, having high severity of chronic illness, and so on. Studies have consistently reported that people are dying by suicide due to unusual COVID-19 fear in Bangladesh and its neighboring countries. ${ }^{1,13,14}$ Therefore, fear of COVID-19 related to the emergence of new variants, unending pandemic situation, and frequent lockdowns, and so on, leads people to an increased risk of psychological suffering, ${ }^{15,16}$ including suicidality. ${ }^{17}$
A recent systematic review considering the COVID-19 pandemic-related mental health problems in South Asian countries found that Bangladeshi people are at higher risk. ${ }^{18}$ Based on recent review studies, mental health problems such as anxiety, frustration, depression, stress, hopelessness, panic, fear, sleep problems, and so on, among the Bangladeshi people are a matter of concern. ${ }^{16,18,19}$ Moreover, as stated above, people with psychological issues are prone to suicidality. ${ }^{20}$ Hence, during the COVID-19 pandemic, the Bangladeshi people may be at a higher risk of suicide due to being susceptible to a higher degree of mental health issues. ${ }^{17}$ But, there are fewer epidemiological studies conducted in the country considering extreme psychological conditions like suicidal behaviors. More specifically, only four studies were conducted in the country, whereas the prevalence of suicidal ideation was found ranging within $5 \%$ to $19 \%$ across different cohorts. ${ }^{21-24}$ Recently, in a systematic review of these studies, Mamun ${ }^{17}$ has identified that the COVID-19 pandemic related suicidal ideation rate tends to increase over time (that is, the more time to implement a survey from pandemic inception, the more suicidal ideation rate will be). Therefore, with the present study being conducted approximately 1 year after pandemic inception, the suicidal ideation rate is anticipated to be higher than prior studies.

Of the four Bangladeshi studies, only one study assessed suicidal ideation by utilizing a scale, Suicide Behaviors Questionnaire-Revised; ${ }^{24}$ and others used a binary item. ${ }^{21-23}$ Where identifying suicidal ideation by a binary response (yes/no) can be limited because this assessment does not provide any severity of suicidality. ${ }^{17}$ Therefore, how many people are actually at greater risk of suicidality (for example, a suicide plan and suicide attempt) is not possibly retrievable from these studies. It should be noted that the suicide completion hierarchy starts with suicidal ideation as the first step, where some of the suicide ideators move forward to the second step (suicide plan), and, finally, a tiny portion of these risky people attempt suicide. ${ }^{25,26}$ Suicidality is an umbrella term that includes suicidal self-injurious behaviors including suicidal ideation, suicide attempt, and suicide plan, where suicide refers to the completion of the process. ${ }^{27}$ However, as most of the prior studies in the country failed to provide information about suicidality hierarchy, this study tried to investigate suicidality by assessing with a different tool.

Finally, the present study objectives include identifying the COVID-19 related (i) suicidality prevalence rates, (ii) 
its associated factors, and (iii) the predictive models explaining its variance. There are three possible prevalence rates for suicidality (suicidal ideation, suicide attempt, and suicide plan), which is tried to observe during the second wave of the pandemic among Bangladeshi young adults in this study. Besides, socio-demographics, behavior and health-related variables, COVID-19 risk, fear of COVID-19, and mental health problems are considered for investigating the predictive models of suicidality related to the COVID-19 pandemic.

\section{Methods}

\section{Study Procedure and Participants}

A web-based cross-sectional study utilizing snowball sampling technique was conducted among Bangladeshi young adults between April 1 and 13, 2020 during the second wave of the pandemic. The survey questionnaire was in Bangla which is the native language of both the participants and researchers. The survey link was circulated across social media platform (ie, Facebook, and WhatsApp) to collect data. The sample size required for the study was calculated based on the following formula which yielded a total of 385, whereas for observational study 500 was suggested as very good sample size according to another study. ${ }^{28}$

$$
\text { Sample Size }=\frac{\frac{z^{2} p(1-p)}{e^{2}}}{1+\frac{z^{2} p(1-p)}{e^{2} N}}
$$

[Here: $\mathrm{N}=$ population size, infinite; $\mathrm{e}=$ Margin of error, $0.05 ; \mathrm{z}=\mathrm{z}$-score, 1.96 (95\% confidence level)]

Approximately a total of 782 data were collected from the survey. After removing the incomplete responses, 756 data were finalized for the analysis. Inclusion criteria included being a young adult, using a popular social media platform, being Bangladeshi, and being willing to participate in study, whereas participants were excluded if they were not from Bangladesh.

\section{Measures}

\section{Sociodemographic Factors}

This survey collected basic socio-demographic information such as age, gender, marital status, religion, educational qualification, socioeconomic status, profession, current residence status, and so on. Socio-economic status was categorized into three groups (ie, lower class, middle class, and higher class - based on an interval of 15,000 Bangladeshi Taka (BDT) monthly family income). In addition, because of overwhelming response from younger students, (i) educational status (up to higher secondary and tertiary education), and (ii) occupation (students and others) were categorized into two types.

\section{Behavioral Health-Related Questions}

Participants' behavior related information related to smoking, drug use (consuming alcohol or using other illicit substances like cannabis, Yaba, etc.), and physical exercise (performing at least $30 \mathrm{~min}$ of physical exercise daily) were collected. Perceived health status was examined using a 5-point Likert scale (very good to very bad), whereas self-rated comorbidity (ie, asthma, hypertension, cardiovascular disease, heart disease, cardiovascular disease, cancer, diabetes, and others) was assessed using a binary response.

\section{COVID-19 Risk-Related Questions}

Participants COVID-19 risk were assessed based on three questions such as participants' infection with COVID-19, participants' friends or family infected with COVID-19, and death of their friends and family. All the responses were in binary and summing up these responses a continuous variable (score ranges from 0 to 3 ) was yielded where higher score indicated higher risk of COVID-19.

\section{Fear of COVID-19 Scale}

Fear of COVID-19 was assessed using the Fear of COVID19 Scale (FCV-19S). ${ }^{29,30}$ The FCV-19S comprises a total of 7 items (eg, "I am afraid of losing my life because of coronavirus-19") score ranging from 7 to 35 based on a 5-point Likert scale (1=strongly disagree, to $5=$ strongly agree). Higher score in the scale indicated a greater Fear of COVID-19. Cronbach's alpha was 0.88 in the present study.

\section{Mental Health Outcomes}

Participants mental health was examined using the Patient Health Questionnaire 4 (PHQ-4) which consist ofa 2-item depression scale (PHQ-2; eg, "Little interest or pleasure in doing things") and a 2-item anxiety scale (GAD-2; eg, "Feeling nervous, anxious or on edge"). ${ }^{31}$ Each items' responses were recorded on a 4 -point Likert scale $(0=$ not at all, to $3=$ nearly every day). The total PHQ-2 and GAD-2 sum scores range from 0 to 6 , whereas a score of $\geq 3$ was used for cutoff points for both depression and anxiety. Cronbach's alpha was 0.69 in the present study.

\section{Suicidal Behaviors}

Suicidal behavior or suicidality were assessed by the last item of the Montgomery-Asberg Depression Rating 
Scale. ${ }^{32}$ The following instruction was given for rating suicidality of the participants:

Feeling that life is not worth living, that a natural death would be welcome, suicidal thoughts, and the preparations for suicide; suicidal attempts should not in themselves influence the rating.

The item was rated on a 7-point Likert scale based on the scale scoring criteria (score range: 0 to 6 ), whereas the higher scores indicate higher suicidality. From the item, having at least symptoms of "frustrated about life and suicidal thoughts float in the head" and "above" were used as cutoff point for suicidality.

\section{Ethical Consideration}

Initially, the CHINTA Research Bangladesh research team developed and approved the project. An IRB approval from the Institute of Allergy and Clinical Immunology of Bangladesh, Savar, Dhaka, Bangladesh, was taken (Reference: IRBIACIB/CEC/03202032). An online consent form was required for participating in this study. Other ethical issues (eg, purpose of the study, participant's right to decline to participate or withdraw from participation, data confidentiality, etc.) were followed as per the Helsinki Declaration of 2013 as suggested by the World Medical Association. ${ }^{33}$

\section{Statistical Analysis}

Data were analyzed using the Statistical Package for Social Science (SPSS) version 25. Before conducting the formal analysis, data were cleaned by Microsoft Excel 2019. Descriptive statistics (ie, percentage, frequency, mean, and standard deviation), independent sample $t$-test, analysis of variance test (ANOVA), and Pearson correlation tests were performed. In addition, multiple hierarchical regression analysis was conducted utilizing suicidality as the dependent variable. Normality and multicollinearity (ie, Condition index, Eigen value, VIF, and tolerance test) of the data were investigated in the present study. The Gaussian assumption was used to assess normality based on the Shapiro-Wilks's test and histograms. Data were considered as high multicollinearity problems if they had Condition index $>15$, Eigen value close to zero, VIF $>10$, and Tolerance $<0.2$. A $p$-value $<0.05$ was set to be statistically significant.

\section{Results}

\section{Characteristics of the Participants}

In the total sample $(\mathrm{N}=756)$, under half of the participants (47.9\%) were within 21 and 23 years, and more than half of them were male $(59 \%)$. The mean age of the participants was reported as $22.24( \pm 4.39)$ years. The majority of the respondents were unmarried (90.6\%), Muslim (89.4\%), students (82.7\%), and urban residents (64.8\%) (Table 1). About $87.8 \%$ and $98.1 \%$ of participants were not involved with smoking and drug use during the COVID-19 pandemic, whereas $54.0 \%$ were not engaged with physical exercise (Table 2). About $6.0 \%$ had a history of personal infection, whereas $42.9 \%$ and $12.8 \%$ had reported having someone of their family members or friends being infected and died of the virus, respectively (Table 3 ). The findings also revealed that the minority of respondents, $35.1 \%$ and $24.1 \%$ experienced depression and anxiety, respectively (Table 4). At the same time, $8.2 \%$ of the participants reported having the symptoms of at least suicidal thoughts (that is, $7.40 \%$ of the participants had only suicidal ideation, whereas $0.10 \%$ of them planned for suicide, and $0.70 \%$ had attempted suicide).

\section{Distribution of the Suicidality Scores}

Tables 1-4 report the associations between suicidality and the studied variables. None of the socio-demographic variables were significantly associated with suicidality (Table 1). Drug users were more prone to suicidality than non-users (2.29 \pm 0.09 vs $1.55 \pm 0.87 ; \mathrm{t}=2.991, p=0.003)$. Besides, the participants who reported worsening their perceived health status were in a trend of reporting higher suicidality scores $(\mathrm{F}=12.958, p<0.001)$. Suicidal behavior was higher in these people suffering from chronic diseases $(1.70 \pm 1.01$ vs $1.52 \pm 0.86 ; \mathrm{t}=2.527, p=0.012)$ (Table 2$)$. Although the personal history of COVID-19 infection was not significantly associated with suicidality, the participants reporting either infection or death history of their family members or friends were at greater risk $(\mathrm{t}=3.339$, $p<0.001$ and $\mathrm{t}=2.871, p=0.004$, respectively) (Table 3 ). In addition, mental health problems such as depression and anxiety were also reported increasing the suicidality mean score $(\mathrm{t}=6.312, p<0.001$ and $\mathrm{t}=8.569, p<0.001$, respectively) (Table 4).

\section{Correlation of the Suicidality}

Table 5 presents correlations between continuous variables and suicidality. There was a significant positive correlation 
Table I Association Between Socio-Demographic Variables and Suicidality

\begin{tabular}{|c|c|c|c|c|}
\hline \multirow[t]{2}{*}{ Variables } & \multirow[t]{2}{*}{ Total Sample; n, \% } & \multicolumn{3}{|c|}{ Suicidality } \\
\hline & & Mean \& SD & F/t Test value & $p$-value \\
\hline \multicolumn{5}{|l|}{ Age (years) } \\
\hline$\leq 20$ & $228,30.2 \%$ & $1.58 \pm 1.04$ & $0.47 \mid$ & 0.624 \\
\hline $21-23$ & $362,47.9 \%$ & $1.54 \pm 0.76$ & & \\
\hline$\geq 24$ & $166,22.0 \%$ & $1.61 \pm 1.02$ & & \\
\hline \multicolumn{5}{|l|}{ Gender } \\
\hline Female & $310,41 \%$ & $1.63 \pm 0.92$ & 1.468 & 0.142 \\
\hline Male & $446,59 \%$ & $1.53 \pm 0.90$ & & \\
\hline \multicolumn{5}{|l|}{ Marital status } \\
\hline Unmarried & $685,90.6 \%$ & $1.55 \pm 0.89$ & -1.191 & 0.234 \\
\hline Married & $71,9.4 \%$ & $1.69 \pm 1.08$ & & \\
\hline \multicolumn{5}{|l|}{ Religion } \\
\hline Muslim & $676,89.4 \%$ & $1.56 \pm 0.89$ & -0.597 & 0.551 \\
\hline Hindu & $80,10.4 \%$ & $1.62 \pm 1.05$ & & \\
\hline \multicolumn{5}{|l|}{ Educational status } \\
\hline Up to secondary & I5I, $20 \%$ & $1.64 \pm 1.03$ & 1.129 & 0.259 \\
\hline More than secondary & $605,80 \%$ & $1.55 \pm 0.88$ & & \\
\hline \multicolumn{5}{|l|}{ Socio-economic status } \\
\hline Lower class & $189,27.2 \%$ & $1.57 \pm 0.94$ & 0.197 & 0.821 \\
\hline Middle class & $237,34.1 \%$ & $1.55 \pm 0.93$ & & \\
\hline Higher class & $270,38.8 \%$ & $1.60 \pm 0.91$ & & \\
\hline \multicolumn{5}{|l|}{ Occupation } \\
\hline Student & $625,82.7 \%$ & $1.57 \pm 0.91$ & 0.689 & 0.888 \\
\hline Non-student & $131,17.3 \%$ & $1.56 \pm 0.95$ & & \\
\hline \multicolumn{5}{|l|}{ Current residence } \\
\hline Rural & $266,35.2 \%$ & $1.54 \pm 0.85$ & -0.580 & 0.562 \\
\hline Urban & $490,64.8 \%$ & $1.58 \pm 0.95$ & & \\
\hline
\end{tabular}

of total comorbidities ( $\mathrm{r}=0.091, p<0.05)$, COVID-19 risk $(\mathrm{r}=0.136, p<0.05)$, fear of COVID-19 $(\mathrm{r}=0.143, p<0.001)$, depression $(\mathrm{r}=0.246 . \quad p<0.001)$ and anxiety $(\mathrm{r}=0.349$, $p<0.001)$ with suicidality.

\section{Predictive Factors of the Suicidality}

Table 6 presents a multiple hierarchical regression analysis with a total of 3 models for predicting suicidality. The first model includes only sociodemographic variables, whereas model 2 compiled health-related variables along with sociodemographic information. In the final model, COVID-19 risk, fear of COVID-19, depression, and anxiety were additionally added. However, all the models were significant except the first model. The final model showed a variance of $17.1 \%$ to predict the participants' suicidality.

\section{Discussion}

The present study was conducted among young Bangladeshi adults during the second wave of the COVID-19 pandemic. The results revealed that about $8.2 \%$ had suicidality, whereas drug use, physical inactivity, poor perceived health status, comorbid symptoms, friends and family COVID-19 infection and death, depression, 
Table 2 Association Between Behavior and Health-Related Variables and Suicidality

\begin{tabular}{|c|c|c|c|c|}
\hline \multirow[t]{2}{*}{ Variables } & \multirow[t]{2}{*}{ Total Sample; n, \% } & \multicolumn{3}{|c|}{ Suicidality } \\
\hline & & Mean \& SD & $F / t$ Test value & $p$-value \\
\hline \multicolumn{5}{|l|}{ Smoking status } \\
\hline Yes & $92,12.2 \%$ & $1.56 \pm 1.03$ & -0.025 & 0.980 \\
\hline No & $664,87.8 \%$ & $1.57 \pm 0.89$ & & \\
\hline \multicolumn{5}{|l|}{ Drug use } \\
\hline Yes & $14,1.9 \%$ & $2.29 \pm 0.09$ & 2.991 & 0.003 \\
\hline No & $742,98.1 \%$ & $1.55 \pm 0.87$ & & \\
\hline \multicolumn{5}{|l|}{ Physical exercise } \\
\hline Yes & $348,46.0 \%$ & $1.48 \pm 0.77$ & -2.447 & 0.015 \\
\hline No & $408,54.0 \%$ & $1.64 \pm 1.01$ & & \\
\hline \multicolumn{5}{|l|}{ Perceived health status } \\
\hline Very Good & $117,15.5 \%$ & $1.43 \pm 1.05$ & 12.958 & $<0.001$ \\
\hline Good & $425,56.2 \%$ & $1.40 \pm 6.72$ & & \\
\hline Neither good nor bad & $184,24.3 \%$ & $1.77 \pm 0.98$ & & \\
\hline Bad & $27,3.6 \%$ & $2.26 \pm|.5|$ & & \\
\hline Very Bad & $3,0.4 \%$ & $3.67 \pm 1.53$ & & \\
\hline \multicolumn{5}{|l|}{ Comorbidity status } \\
\hline Yes & $206,27.2 \%$ & $1.70 \pm 1.01$ & 2.527 & 0.012 \\
\hline No & $550,72.8 \%$ & $1.52 \pm 0.86$ & & \\
\hline
\end{tabular}

Table 3 Association Between COVID Risk-Related Variables and Suicidality

\begin{tabular}{|c|c|c|c|c|}
\hline \multirow[t]{2}{*}{ Variables } & \multirow[t]{2}{*}{ Total Sample; n, \% } & \multicolumn{3}{|c|}{ Suicidality } \\
\hline & & Mean \& SD & $F / t$ Test value & $p$-value \\
\hline \multicolumn{5}{|c|}{ Personal COVID-I9 infection } \\
\hline No & $711,94.0 \%$ & $1.56 \pm 0.88$ & -1.090 & 0.276 \\
\hline Yes & $45,6.0 \%$ & $1.71 \pm 1.31$ & & \\
\hline \multicolumn{5}{|c|}{ Friend and family COVID- 19 infection } \\
\hline No & $432,57.1 \%$ & $1.47 \pm 0.78$ & -3.339 & 0.001 \\
\hline Yes & $324,42.9 \%$ & $1.69 \pm 1.05$ & & \\
\hline \multicolumn{5}{|c|}{ Friend and family COVID- 19 death } \\
\hline No & $659,87.2 \%$ & $1.53 \pm 0.87$ & -2.871 & 0.004 \\
\hline Yes & $97,12.8 \%$ & $1.81 \pm 1.15$ & & \\
\hline
\end{tabular}

and anxiety were the associated factors of suicidality. However, it is alleged that the suicide mortality rate increases during any outbreaks like the current COVID19 outbreak. $^{34}$ Subsequently, a few studies concerning completed suicide cases were reported from Bangladesh. ${ }^{1,10,11,35}$ But the country still lacks epidemiological studies assessing suicidal behaviors (as opposed to complete suicide), where this finding may help in public policy formulation and practice.

Several studies have reported psychological impact of confinement and lockdown during the COVID-19 pandemic. ${ }^{18}$ However, the first study on suicidal ideation conducted within April 2020, among a nationwide sample, identified the prevalence rate to be $5 \%,{ }^{21}$ whereas the rate 
Table 4 Association Between Mental Health Problems and Suicidality

\begin{tabular}{|l|l|l|l|l|}
\hline \multirow{2}{*}{ Variables } & Total Sample; n, \% & \multicolumn{3}{|c|}{ Suicidality } \\
\cline { 3 - 5 } \multicolumn{2}{|l|}{} & Mean \& SD & t Test value & p-value \\
\hline Depression & $491,64.9 \%$ & $1.42 \pm 0.73$ & -6.312 & $<0.001$ \\
\hline $\begin{array}{l}\text { No } \\
\text { Yes }\end{array}$ & $265,35.1 \%$ & $1.84 \pm 1.12$ & \\
\hline Anxiety & $574,75.9 \%$ & $1.40 \pm 1.74$ & -8.569 & $<0.001$ \\
\hline No & $182,24.1 \%$ & $2.05 \pm 1.19$ & \\
\hline
\end{tabular}

Table 5 Pearson Correlation Coefficients Between Continuous Variables and Suicidality

\begin{tabular}{|c|c|c|c|c|c|c|c|c|c|}
\hline Variables & Mean \& SD & I & 2 & 3 & 4 & 5 & 6 & 7 & 8 \\
\hline Suicidality (I) & $1.57 \pm 0.91$ & I & & & & & & & \\
\hline Age (2) & $22.24 \pm 4.39$ & 0.007 & 1 & & & & & & \\
\hline Income (3) & $41851.04 \pm 82303.58$ & 0.033 & 0.059 & I & & & & & \\
\hline Total comorbidities (4) & $0.29 \pm 0.50$ & $0.09 I^{*}$ & $0.200 * *$ & 0.032 & I & & & & \\
\hline COVID-19 risk (5) & $0.62 \pm 0.762$ & $0.136^{*}$ & $0.101 * *$ & $0.169 * *$ & 0.037 & I & & & \\
\hline Fear of COVID-19 (6) & $17.63 \pm 5.88$ & $0.143^{* *}$ & 0.012 & -0.016 & 0.008 & $0.082 *$ & I & & \\
\hline Depression (7) & $2.15 \pm 1.19$ & $0.246 * *$ & -0.028 & 0.014 & $0.083^{*}$ & $0.108 * *$ & $0.183^{*} *$ & I & \\
\hline Anxiety (8) & $1.78 \pm 1.52$ & $0.349 * *$ & $-0.076^{*}$ & 0.046 & $0.137^{* *}$ & $0.159 * *$ & $0.322 * *$ & $0.560 * *$ & I \\
\hline
\end{tabular}

Notes: $* *<0.01 . *<0.05$.

increased to $6.1 \%$ in another study while the survey was conducted a few days later. ${ }^{22}$ Later on, the prevalence of suicidal ideation was observed to increase over time; more specifically, $12.8 \%$ was reported in a study from April to May $2020,{ }^{23}$ which further increased to $19 \%$ in another study conducted within July $2020 .^{24}$ In the present study conducted 1 year after the pandemic inception, the suicidal ideation rate was expected to be higher than the prior studies; but unexpectedly, the rate was lower. About $8.2 \%$ of the participants reported having the symptoms of at least suicidal thoughts (that is, $7.40 \%$ of the participants had only suicidal ideation, whereas $0.10 \%$ planned for suicide $0.70 \%$ had attempted suicide).

This fluctuation in suicidality prevalence rate may be due to the use of different instruments and/or different timeframes to estimate prevalence rates within nonrepresentative samples. It should be noted that the first three studies used a single item for assessing suicidal ideation with a binary response (Yes/No), and Rahman et $\mathrm{al}^{24}$ used 4-item Suicide Behaviors QuestionnaireRevised (SBQ-R). But it was a single item from the Montgomery-Asberg Depression Rating Scale used in the present study. Disparities of the studied cohorts (eg, university students, health-care professionals, general population), and sampling technique, participant characteristics, and so on, across these studies might also affect the suicidality rate. Concludingly, it can be stated that a large portion of the present sample is still at risk of suicide, whom should still be facilitated with mental health support and services to reduce further suicide occurrences.

It is well established that males commit more suicide, where females are at a higher risk of attempting suicide. As per the WHO, a 13.7 per 100,000 population rate of male suicide was reported globally, whereas it was 7.5 per 100,000 for females. ${ }^{36}$ Furthermore, several studies observe the diversity of suicide and suicidal behaviors across gender, including attributing factors such as social construction of masculinity and femininity, life historyrelated issues, lifestyle and social status conditions, their 
Table 6 Predictive Models for the Suicidality

\begin{tabular}{|c|c|c|c|c|c|c|c|c|c|c|c|c|}
\hline \multirow[t]{3}{*}{ Variables } & \multicolumn{4}{|c|}{ Model I } & \multicolumn{4}{|c|}{ Model 2} & \multicolumn{4}{|c|}{ Model 3} \\
\hline & \multicolumn{4}{|c|}{$\begin{array}{l}{\left[R^{2}=0.008, F=0.726, \text { Adjusted } R^{2}=\right.} \\
-.003, p=0.668]\end{array}$} & \multicolumn{4}{|c|}{$\begin{array}{l}{\left[R^{2}=0.079, F=4.486, \text { Adjusted }\right.} \\
\left.R^{2}=0.061, p<0.001\right]\end{array}$} & \multicolumn{4}{|c|}{$\begin{array}{l}{\left[R^{2}=0.171, F=8.245, \text { Adjusted }\right.} \\
\left.R^{2}=0.151, p<0.001\right]\end{array}$} \\
\hline & B & SE & $\boldsymbol{\beta}$ & $p$ & B & SE & $\boldsymbol{\beta}$ & $p$ & B & SE & $\boldsymbol{\beta}$ & $p$ \\
\hline Constant & 1.644 & 0.329 & & 0.000 & 2.203 & 0.623 & & 0.000 & 1.974 & 0.596 & & 0.001 \\
\hline Age & 0.002 & 0.010 & 0.010 & 0.846 & 0.000 & 0.010 & 0.001 & 0.991 & 0.004 & 0.010 & 0.017 & 0.709 \\
\hline Gender $^{\mathrm{a}}$ & -0.076 & 0.073 & -0.040 & 0.299 & 0.035 & 0.076 & 0.019 & 0.648 & 0.124 & 0.074 & 0.066 & 0.096 \\
\hline Marital Status ${ }^{b}$ & 0.134 & 0.143 & 0.043 & 0.348 & 0.113 & 0.138 & 0.036 & 0.414 & 0.089 & 0.132 & 0.028 & 0.502 \\
\hline Religion $^{c}$ & 0.095 & 0.113 & 0.032 & 0.402 & 0.055 & 0.110 & 0.019 & 0.615 & 0.052 & 0.106 & 0.017 & 0.626 \\
\hline Education Level $^{d}$ & -0.115 & 0.089 & -0.049 & 0.200 & -0.078 & 0.087 & -0.034 & 0.367 & -0.053 & 0.083 & -0.023 & 0.523 \\
\hline Income & $<0.001$ & 0.000 & 0.035 & 0.367 & $<0.001$ & 0.000 & 0.025 & 0.504 & $<0.001$ & 0.000 & 0.008 & 0.819 \\
\hline Residence $^{\mathrm{e}}$ & 0.018 & 0.075 & 0.009 & 0.812 & 0.019 & 0.073 & 0.010 & 0.796 & -0.027 & $0.07 I$ & -0.014 & 0.706 \\
\hline Occupation $^{f}$ & -0.068 & 0.114 & -0.028 & 0.550 & -0.080 & 0.111 & -0.033 & 0.473 & -0.049 & 0.106 & -0.020 & 0.644 \\
\hline Health Status & & & & & 0.266 & 0.050 & 0.210 & 0.000 & 0.145 & 0.049 & 0.115 & 0.003 \\
\hline Multi comorbidities & & & & & 0.068 & 0.070 & 0.038 & 0.330 & 0.029 & 0.067 & 0.016 & 0.663 \\
\hline Smoking status ${ }^{g}$ & & & & & 0.149 & 0.114 & 0.054 & 0.191 & 0.191 & 0.109 & 0.069 & 0.080 \\
\hline Drug use ${ }^{g}$ & & & & & -0.888 & 0.260 & -0.135 & 0.001 & -1.011 & 0.248 & -0.154 & 0.000 \\
\hline Physical exercise $^{g}$ & & & & & 0.133 & 0.070 & 0.072 & 0.058 & 0.074 & 0.067 & 0.040 & 0.270 \\
\hline COVID-19 risk & & & & & & & & & 0.090 & 0.046 & 0.074 & 0.049 \\
\hline Fear of COVID-19 & & & & & & & & & 0.006 & 0.006 & 0.039 & 0.309 \\
\hline Depression & & & & & & & & & 0.067 & 0.033 & 0.086 & 0.043 \\
\hline Anxiety & & & & & & & & & 0.148 & 0.029 & 0.243 & 0.000 \\
\hline
\end{tabular}

Notes: ${ }^{\mathrm{a}} \mathrm{I}=$ Female, 2 = Male; ${ }^{\mathrm{b}} \mathrm{I}=$ Unmarried, 2 = Married; ${ }^{\mathrm{c}} \mathrm{I}=$ Muslim, 2 = Hindu; ${ }^{\mathrm{d}} \mathrm{I}=$ Up to secondary, 2 = More than secondary; ${ }^{\mathrm{e}} \mathrm{I}=$ Rural, $2=$ Urban; ${ }^{\mathrm{f}} \mathrm{I}=$ Students, 0 $=$ Non-students; ${ }^{g}$ I $=$ Yes, 2 = No.

role in society, and psychological vulnerabilities. ${ }^{37-40}$ Consistent with this scenario, all the prior studies conducted during the pandemic reported females were at higher risk of suicidal ideation ${ }^{21-24}$ and other mental health problems, ${ }^{41,42}$ but no gender-based statistical difference was found in this study. Such an unexpected finding needs to be explored in future studies considering a greater focus on gender-based vulnerabilities towards suicide.

In a prior Bangladeshi study during the pandemic, smokers were reported to be at an increased risk of suicidal ideation, ${ }^{23}$ although no significant association was found in this study. In contrast, Mamun et $\mathrm{al}^{21}$ detected alcohol consumption as one of the risk factors of suicidal ideation, which is consistent with this study. Using drugs is more frequent among the individuals with mental health problems, trouble in the relationship, social life, and other difficulties, which are the common attributing factors of poor self-care and mental health problems. ${ }^{43-45}$ These people may be reckless about their health and selfcaring; thus, drug addicts are less concerned about keeping themselves fit by avoiding unhealthy behaviors (eg, drug use) and engaging with regular physical activities. Similarly, lack of physical activity increases the risk of mental health problems along with suicide-related outcomes reported in prior studies, ${ }^{46}$ as consistent in this study. These individuals are suffering from mental health conditions and are globally being reported to be at a greater risk of suicide. ${ }^{20}$ For instance, a 13.81-fold 
higher risk of suicidal ideation was reported for depression in a Bangladeshi study conducted during the pandemic, whereas it was 6.45- and 9.53-fold for anxiety and stress, respectively. $^{23}$ Therefore, caution should be made to increase mental health support for individuals with unhealthy lifestyles, mental disorders, and so on, to minimize suicidal risk.

For the first time, this study provides any predicting model for suicidality among young adults during the COVID-19 pandemic in the context of Bangladesh. All the models were significant except the first model, whereas only sociodemographic variables were used. Sociodemographic and behavior and health-related factors significantly predicted about $7.9 \%$ suicidality variance, whereas an additional $9.2 \%$ variance is added while the final model consisted COVID-19 risk, fear of COVID-19, and mental health issues (ie, depression and anxiety). However, as per the prior outbreaks like the COVID-19 outbreak, fear of infection was found as the prominent suicide stressors along with social isolation-related issues, difficulties in everyday life activities, the burden of longterm illnesses, and so on. ${ }^{47,48}$ Participants in the study, with a history of family members' and friends' infection and/or death, were more likely to report higher suicidality. Besides this, although the personal history of being infected with COVID-19 was not significantly associated with a higher tendency of suicidal behavior, fear of COVID-19 infection was positively correlated with suicidality. This may be because people thinking more about their vulnerabilities toward virus infection are at greater risk of uncertainty and phobia. As reflected by Mamun et $\mathrm{al}^{21}$ findings, the COVID-19 related knowledge had a significant role in predicting the fear of COVID-19, which further subsequently and significantly contribute to higher preventive behaviors and mental health issues. Therefore, community-based health education, awarenessrelated strategies to increase knowledge about COVID-19 can be a probable solution to reduce the fear of COVID19 , by which unwanted mental health instabilities can be suppressed.

The generalizability of this study's findings can be limited due to its study nature that is being online crosssectional, along with selection bias (snowball), social desirability bias, and so on, since its accessibility is linked to its own internet connection and social group. In addition, because of online data collection, most participants belonged to educated younger age, which may limit the study findings' generalizability. Since all responses are subjective, and all subjects completed the survey at one timepoint, so the findings are subject to recall bias. For assessing physical exercise, a single question on whether they performed daily $30 \mathrm{~min}$ was asked despite considering exercise type, severity, number of days in a week, and so on, which may be a weak indicator. The study did not assess other important factors related to mental health problems, such as economic hardship, financial threatrelated factors, and exposure to screen time during the pandemic. $^{17,49}$ In addition, information related to stigma was not considered in this study, which may influence lifestyle, behaviors, and quality of life including suicidality. Therefore, considering these limitations, further studies with rigorous methodologies from a representative sample are required. Despite these limitations, the present study provides some baseline information regarding suicidality in the context of the second wave of the COVID19 pandemic in Bangladesh.

\section{Conclusions}

Although the present study reported a lower severity of suicidality than prior studies conducted during the first wave of the pandemic, a larger portion of Bangladeshi young adults are still at risk of suicide whom to be given attention. This study observes higher suicidality rates among young adults with unhealthy lifestyle (eg, drug using, performing less exercise), mental health-related suffering (eg, depression and anxiety), COVID-19 infection risk, and fear of COVID-19. These findings can be beneficial to other researchers in understanding youth suicidality and implementing any mental health initiatives to prevent further suicide occurrences.

\section{Acknowledgments}

The authors also like to acknowledge that their affiliation, the CHINTA Research Bangladesh, was formerly known as the Undergraduate Research Organization. It should be noted that from this project, a manuscript with different study objective is published. ${ }^{50}$

\section{Funding}

The present study did not get any financial support.

\section{Disclosure}

The authors reported no conflicts of interest in this work. 


\section{References}

1. Mamun MA, Griffiths MD. First COVID-19 suicide case in Bangladesh due to fear of COVID-19 and xenophobia: possible suicide prevention strategies. Asian J Psychiatr. 2020;51:e102073. doi:10.1016/j.ajp.2020.102073

2. Anwar S, Nasrullah M, Hosen MJ. COVID-19 and Bangladesh: challenges and how to address them. Front Public Heal. 2020;8:154. doi:10.3389/fpubh.2020.00154.

3. López-Bueno R, Calatayud J, Ezzatvar Y, et al. Association between current physical activity and current perceived anxiety and mood in the initial phase of COVID-19 confinement. Front Psychiatry. 2020;11:729. doi:10.3389/fpsyt.2020.00729.

4. Tornaghi M, Lovecchio N, Vandoni M, Chirico A, Codella R. Physical activity levels across COVID-19 outbreak in youngsters of Northwestern Lombardy. $J$ Sports Med Phys Fitness. 2021;61:971-976. doi:10.23736/S0022-4707.20.11600-1

5. Codella R, Chirico A, Lucidi F, Ferrulli A, La Torre A, Luzi L. The immune-modulatory effects of exercise should be favorably harnessed against COVID-19. J Endocrinol Invest. 2020;1:1119-1122. doi:10.1007/s40618-020-01403-5.

6. Chirico A, Lucidi F, Galli F, et al. COVID-19 outbreak and physical activity in the Italian population: a cross-sectional analysis of the underlying psychosocial mechanisms. Front Psychol. 2020;11:1-13. doi:10.3389/fpsyg.2020.02100

7. Brooks SK, Webster RK, Smith LE, et al. The psychological impact of quarantine and how to reduce it: rapid review of the evidence. Lancet. 2020;395:912-920. doi:10.1016/S0140-6736(20)30460-8

8. Hossain MM, Sultana A, Purohit N. Mental health outcomes of quarantine and isolation for infection prevention: a systematic umbrella review of the global evidence. Epidemiol Health. 2020;42: e2020038. doi:10.4178/epih.e2020038

9. Banna H. Minimising the economic impact of Coronavirus in Bangladesh. Bus Stand; 2020. Available from: https://www.tbsnews. net/thoughts/minimising-economic-impact-coronavirus-bangladesh -56449. Accessed May 13, 2020.

10. Bhuiyan AKMI, Sakib N, Pakpour AH, Griffiths MD, Mamun MA. COVID-19-related suicides in Bangladesh due to lockdown and economic factors: case study evidence from media reports. Int J Ment Health Addict. 2020. doi:10.1007/s11469-020-00307-y

11. Boshra SN, Islam MM, Griffiths MD. The demography and apparent risk factors of COVID-19-related suicides in Bangladesh in a seven-month period of the pandemic. medRxiv. 2020. doi:10.1101/ 2020.08.11.20171272

12. Leaune E, Samuel M, Oh H, Poulet E, Brunelin J. Suicidal behaviors and ideation during emerging viral disease outbreaks before the COVID-19 pandemic: a systematic rapid review. Prev Med (Baltim). 2020;141:e106264. doi:10.1016/j.ypmed.2020.106264

13. Dsouza DD, Quadros S, Hyderabadwala ZJ, Mamun MA. Aggregated COVID-19 suicide incidences in India: fear of COVID-19 infection is the prominent causative factor. Psychiatry Res. 2020;290:e113145. doi:10.1016/j.psychres.2020.113145

14. Mamun MA, Ullah I. COVID-19 suicides in Pakistan, dying off not COVID-19 fear but poverty?-The forthcoming economic challenges for a developing country. Brain Behav Immun. 2020;87:163-166. doi:10.1016/j.bbi.2020.05.028

15. Quadros S, Garg S, Ranjan R, Vijayasarathi G, Mamun MA. Fear of COVID 19 infection across different cohorts: a scoping review. Front Psychiatry. 2021;12:708430. doi:10.3389/fpsyt.2021.708430

16. Hosen I, al mamun F, Mamun MA. Prevalence and risk factors of mental disorders during the COVID-19 pandemic in Bangladesh: a systematic review. Res $S q$. 2021. doi:10.21203/rs.3.rs-631435/v1

17. Mamun MA. Suicide and suicidal behaviors in the context of COVID-19 pandemic in Bangladesh: a systematic review. Psychol Res Behav Manag. 2021;14:695-704. doi:10.2147/PRBM.S315760
18. Hossain MM, Rahman M, Trisha NF, et al. Prevalence of anxiety and depression in South Asia during COVID-19: a systematic review and meta-analysis. Heliyon. 2020;7:e06677. doi:10.1016/j.heliyon.2021. e06677

19. al Mamun F, Hosen I, Misti JM, Kaggwa MM, Mamun MA. Mental disorders of Bangladeshi students during the COVID-19 pandemic: a systematic review. Psychol Res Behav Manag. 2021;14:645-654. doi:10.2147/PRBM.S315961

20. Fleischmann A, Bertolote JM, Belfer M, Beautrais A. Completed suicide and psychiatric diagnoses in young people: a critical examination of the evidence. Am J Orthopsychiatry. 2005;75:676-683. doi:10.1037/0002-9432.75.4.676

21. Mamun MA, Sakib N, Gozal D, Bhuiyan AKMI, Hossain S, BodrudDoza M. The COVID-19 pandemic and serious psychological consequences in Bangladesh: a population-based nationwide study. J Affect Disord. 2021;279:462-472. doi:10.1016/j.jad.2020.10.036

22. Mamun MA, Akter T, Zohra F, et al. Prevalence and risk factors of COVID-19 suicidal behavior in Bangladeshi population: are healthcare professionals at greater risk? Heliyon. 2020;6:e05259. doi:10.1016/j.heliyon.2020.e05259

23. Tasnim R, Islam MS, Sujan MSH, Sikder MT, Potenza MN. Suicidal ideation among Bangladeshi university students early during the COVID-19 pandemic: prevalence estimates and correlates. Child Youth Serv Rev. 2020;119:105703. doi:10.1016/j.childyouth.2020.10 5703

24. Rahman ME, Al ZA, Al Mazid Bhuiyan MR, Jobe MC, Ahsan Khan MK. Suicidal behaviors and suicide risk among Bangladeshi people during the COVID-19 pandemic: an online cross-sectional survey. Heliyon. 2021;7:e05937. doi:10.1016/j.heliyon.2021.e05937

25. Rasheduzzaman M, al Mamun F, Faruk MO, Hosen I, Mamun MA. Depression in Bangladeshi university students: the role of sociodemographic, personal, and familial psychopathological factors. Perspect Psychiatr Care. 2021. doi:10.1111/ppc.12722

26. Klonsky ED, May AM, Saffer BY. Suicide, suicide attempts, and suicidal ideation. Annu Rev Clin Psychol. 2016;12:307-330. doi:10.1146/annurev-clinpsy-021815-093204

27. Keefner TP, Stenvig T. Suicidality: an evolutionary concept analysis. Issues Ment Health Nurs. 2021;42:227-238. doi:10.1080/ 01612840.2020 .1793243

28. Bujang MA, Sa'at N, Sidik TMITAB, Joo LC. Sample size guidelines for logistic regression from observational studies with large population: emphasis on the accuracy between statistics and parameters based on real life clinical data. Malays $J$ Med Sci. 2018;25:122-130. doi:10.21315/mjms2018.25.4.12

29. Ahorsu DK, Lin C-Y, Imani V, Saffari M, Griffiths MD, Pakpour AH. The fear of COVID-19 scale: development and initial validation. Int J Ment Health Addict. 2020. doi:10.1007/s11469-020-00270-8

30. Sakib N, Bhuiyan AKMI, Hossain S, et al. Psychometric validation of the bangla fear of COVID-19 scale: confirmatory factor analysis and rasch analysis. Int J Ment Health Addict. 2020. doi:10.1007/ s11469-020-00289-x

31. Löwe B, Wahl I, Rose M, et al. A 4-item measure of depression and anxiety: validation and standardization of the Patient Health Questionnaire-4 (PHQ-4) in the general population. J Affect Disord. 2010;122:86-95. doi:10.1016/j.jad.2009.06.019

32. Montgomery SA, Åsberg M. A new depression scale designed to be sensitive to change. Br J Psychiatry. 1979;134:382-389. doi:10.1192/ bjp.134.4.382

33. Association WM. World medical association declaration of Helsinki: ethical principles for medical research involving human subjects. JAMA. 2013;310:2191-2194. doi:10.1001/jama.2013.281053

34. John A, Okolie C, Eyles E, et al. The impact of the COVID-19 pandemic on self-harm and suicidal behaviour: a living systematic review. F1000Research. 2020;9:e1097. doi:10.12688/f1000rese $\operatorname{arch} .25522 .1$ 
35. Mamun MA, Chandrima RM, Griffiths MD. Mother and son suicide pact due to COVID-19-related online learning issues in Bangladesh: an unusual case report. Int J Ment Health Addict. 2020. doi:10.1007/ s11469-020-00362-5

36. World Health Organization. Suicide in the world: global health estimates; 2019. Available from: https://apps.who.int/iris/handle/ 10665/326948. Accessed May 8, 2021.

37. Göktaş S, Metintaş S. Suicide mortality trends by age, gender and method in Turkey, 2002-2015. Turk $J$ Public Health. 2019;17:195-206. doi:10.20518/tjph.442055

38. Vijayakumar L. Suicide in women. Indian J Psychiatry. 2015;57: S233. doi:10.4103/0019-5545.161484

39. Payne S, Swami V, Stanistreet DL. The social construction of gender and its influence on suicide: a review of the literature. J Mens Health. 2008;5:23-35. doi:10.1016/j.jomh.2007.11.002

40. Kaggwa MM, Muwanguzi M, Nduhuura E, et al. Suicide among Ugandan university students: evidence from media reports for 2010-2020. BJPsych Int. 2021;18(3):63-67. doi:10.1192/bji.2021.13

41. Mattioli AV, Sciomer S, Maffei S, Gallina S. Lifestyle and stress management in women during COVID-19 pandemic: impact on cardiovascular risk burden. Am J Lifestyle Med. 2021;15:356-359. doi:10.1177/1559827620981014

42. Torres SJ, Nowson CA. Relationship between stress, eating behavior, and obesity. Nutrition. 2007;23:887-894. doi:10.1016/j.nut.2007.0 8.008

43. Bradizza CM, Stasiewicz PR, Paas ND. Relapse to alcohol and drug use among individuals diagnosed with co-occurring mental health and substance use disorders: a review. Clin Psychol Rev. 2006;26:162-178. doi:10.1016/j.cpr.2005.11.005
44. Boys A, Marsden J, Strang J. Understanding reasons for drug use amongst young people: a functional perspective. Health Educ Res. 2001;16:457-469. doi:10.1093/her/16.4.457

45. Nabi MH, Masud I, Humaira T, et al. Reasons of relapse and pattern of drug use among the substance users in Bangladesh: a nationwide study. J Ethn Subst Abuse. 2020;1-10. doi:10.1080/15332640.2 020.1801549

46. Grasdalsmoen M, Eriksen HR, Lønning KJ, Sivertsen B. Physical exercise, mental health problems, and suicide attempts in university students. BMC Psychiatry. 2020;20:175. doi:10.1186/s12888-02002583-3

47. Cheung YT, Chau PH, Yip PSF. A revisit on older adults suicides and Severe Acute Respiratory Syndrome (SARS) epidemic in Hong Kong. Int J Geriatr Psychiatry. 2008;23:1231-1238. doi:10.1002/ gps. 2056

48. Yip PSF, Cheung YT, Chau PH, Law YW. The impact of epidemic outbreak: the case of severe acute respiratory syndrome (SARS) and suicide among older adults in Hong Kong. Cris J Cris Interv Suicide Prev. 2010;31:86. doi:10.1027/0227-5910/a000015

49. Smith L, Jacob L, Trott M, et al. The association between screen time and mental health during COVID-19: a cross sectional study. Psychiatry Res. 2020;292:113333. doi:10.1016/j.psychres.202 0.113333

50. Hasan M, Maliha Z, Rahman A, Mamun MA. Insomnia in Bangladeshi young adults during the COVID-19 pandemic: the role of behavioral factors, COVID-19 risk and fear, and mental health issues. Sleep Vigil. 2021. doi:10.1007/s41782-021-00161-5
Risk Management and Healthcare Policy

\section{Publish your work in this journal}

Risk Management and Healthcare Policy is an international, peerreviewed, open access journal focusing on all aspects of public health, policy, and preventative measures to promote good health and improve morbidity and mortality in the population. The journal welcomes submitted papers covering original research, basic science, clinical \& epidemiological studies, reviews and evaluations, guidelines, expert opinion and commentary, case reports and extended reports. The manuscript management system is completely online and includes a very quick and fair peer-review system, which is all easy to use. Visit http://www.dovepress.com/testimonials.php to read real quotes from published authors. 